\title{
Correspondence
}

\section{Combined lumbosacral plexus block}

To the Editor:

Vaghadia et al. ${ }^{1}$ recommended the use of continuous lumbosacral block (CLSPB) instead of a single injection technique. However, some comments are needed. The authors did not emphasize the need for a test dose before injecting the top-up of local anaesthetic, although there is a risk of catheter migration. In addition, two of their patients received large volumes of local anaesthetic with the possible risk of toxicity.

During the last two years, we have performed CLSPB, as described by Winnie, ${ }^{2}$ as the sole anaesthetic technique in 12 cases of below knee (BK) and six cases of above knee (AK) amputation. Failure of the block in three out of 18 cases was due to lack of anaesthesia in the area innervated by the sciatic nerve. All three occurred in patients undergoing $\mathrm{AK}$ amputation which suggests that, in addition, block of the sciatic nerve ought to be considered, when anesthesia of the posterior aspect of the lower limb is needed. ${ }^{3}$ In 13 cases we were unable to elicit paraesthesia because of the patients' altered mental status, and a nerve stimulator was used to identify the plexus.

The sole complication noted was accidental spinal anaesthesia which occurred in four cases. In three we were unable to aspirate cerebrospinal fluid (CSF) and this may have been due to a "ball-valve" effect on account of tissue debris at the tip of the needle. In one of the patients a high spinal block resulted which resolved after two hours of artificial ventilation and fluid resuscitation. This case suggested the use of a test dose before injecting the total dose. Indeed, in the remaining two cases, in which only $3 \mathrm{ml}$ of local anaesthetic was injected, spinal anaesthesia was induced accidentally, but surgery proceeded uneventfully.

In view of these results the following conclusions may be drawn. Sciatic nerve block should be added for anaesthesia of the posterior aspect of the lower limb. Negative aspiration of CSF does not exclude accidental injection of anaesthetic into the spinal space and therefore, a test dose of $3 \mathrm{ml}$ should be administered prior to injection of the full volume of anaesthetic.

\section{Tiberiu Ezri MD}

Peter Szmuk MD

\author{
Virgil Priscu MD \\ David Soroker MD \\ Department of Anesthesiology \\ Kaplan Hospital \\ Affiliated to the Hebrew University \\ and Hadassah, Jerusalem
}

\section{REFERENCES}

1 Vaghadia H, Kapnoudhis $P$, Jenkins $L$, Taylor $D$.

Continuous lumbosacral using a Tuohy needle and catheter technique. Can J Anaesth 1992; 39: 75-8.

2 Winnie AP, Ramamurthy $S$, Durrani $Z$, Radonjic $R$. Plexus block for lower extremity surgery. Anesthesiology Review 1974; 1: 11-6.

3 Parkinson $S K$, Mueller JB, Little $W L$, Bailey $S L$. Extent of blockade with various approaches to the lumbar plexus. Anesth Analg 1989; 68: 243-8.

\section{Finger pulse oximeter response}

To the Editor:

In the recent article published by Ding $e t$ al. ${ }^{1}$ the lag time of a finger probe pulse oximeter response was decreased by the performance of an axillary block. The conclusion of this article was that this lag time is not primarily determined by the pulse oximeter itself, but by the circulation time.

Our group discussed the subject of pulse oximeter lag time in a recently published article on preoxygenation in children. ${ }^{2}$ In our study we observed that $\mathrm{SaO}_{2}$ during a period of apnoea after rapid intravenous induction of anaesthesia. The mean \pm SD time of apnoea for the $\mathrm{SaO}_{2}$ to decrease to $90 \%$ was $92 \pm 21 \mathrm{sec}$ vs $144 \pm$ $35 \mathrm{sec}$ when one minute of preoxygenation was compared with three minutes. Although manual ventilation with $100 \% \mathrm{O}_{2}$ was reinstituted when the $\mathrm{SaO}_{2}$ reached $90 \%$, somewhat surprisingly the $\mathrm{SaO}_{2}$ continued to decrease and its nadir was reached after 10 to $15 \mathrm{sec}$ in all patients. To explain this phenomenon we cited a study done by Wexler et al. in 1946, using a Millikan oximeter, that determined the pulmonary to systemic capillary circulation time after a deep breath of $100 \% \mathrm{~N}_{2}{ }^{3}$

We think that this pioneer study by Wexler $e$ t al. should be cited whenever the subject discussed is pulse oximeter lag time. The analysis of this study would certainly have contributed to the discussion and would corroborate, al- 